\title{
Classification and Searching in Java API Reference Documentation
}

\author{
Monali Metkar \\ K.K.W.I.E.E.R \\ Nashik, \\ University Of Pune, India
}

\author{
S. M. Kamalapur \\ K.K.W.I.E.E.R \\ Nashik, \\ University Of Pune, India
}

\begin{abstract}
Application Program Interface (API) allows programmers to use predefined functions instead of writing them from scratch. Description of API elements that is Methods, Classes, Constructors etc. is provided through API Reference Documentation. Hence API Reference Documentation acts as a guide to user or developer to use API's. Different types of Knowledge Types are generated by processing this API Reference Documentation. And this Knowledge Types will be used for Classification and Searching of Java API Reference Documentation.
\end{abstract}

Keywords: API, API Reference Documentation, Knowledge Types, Classification, Searching.

\section{INTRODUCTION}

An Application Programming Interface (API) is a set of commands, functions, and protocols. It also specifies the interaction between the software components. In most procedural languages, an API specifies a set of functions or routines that accomplish a specific task or are allowed to interact with a specific software component. For example, consider following Constructor in Java:

\section{AbstractAction()}

Whenever user or developer is referring to an API and has planned to use it for specific purpose API Reference documentation works as a guide. API Reference Documentation is an important part of programming with APIs and it complements the API by providing information about the API. So, it plays a crucial role in how developers learn and use an A PI, and developers will expect the information about API elements they should find therein By considering the above example, if new developer wishes to use "AbstractAction()" constructor in Java Program, he can refer to API Reference Documentation of Java and he will find the description of "AbstractAction()" constructor in Constructor Summary as:

Creates an Action.

In above example Java Documentation is considered and Java APIs are documented through Javadocs which is a set of web pages such that one for each package or type in the API.

To enhance the quality of API reference documentation and the efficiency with which the relevant information it contains can be accessed, it's necessary to first understand its contents by analyzing it. Therefore, to reason about the quality and value of Java API reference documentation, focus should be about what knowledge it contains. Because Knowledge refers to retrieve useful information from data and then use this knowledge for specific purpose. By analyzing the contents of Java API Reference Documentation, Knowledge is generated and this knowledge can be categorized further.
Previous work focused separately on Studies of Knowledge Categorization and of API reference Documentation and Knowledge retrieval was done based on Experience, Observations and Analysis.

So proposed system focuses on generation of Knowledge Types, classification of API Reference Document according to Knowledge Types and also on searching depending upon Knowledge Types.

Section 2 focuses on Literature Review. Section 3 gives Implementation Details with Block Diagram, Concept with Example and Algorithms are highlighted in Section 4. Data Set, Results obtained and Performance Measure are discussed in Section 5 of Results. The paper ends with concluding remarks.

\section{LITERATURE REVIEW}

The previous work mainly focused on the Knowledge Categorization and API Reference Documentation Separately.

\subsection{API Reference Documentation}

Study of documentation needs for a domain-specific API, using surveys and interviews of developers was done by Nykaza et al.[6] This study identified, among other requirements and the importance of an overview section in API documentation.

Jeong et al. [10] conducted a lab study with eight participants to assess the documentation of a specific service-oriented architecture. This study identified 18 guidelines they believe would lead to increased documentation quality for the system under study, including "explaining starting points" for using the API.

Robillard and DeLine [9] identified the obstacles faced by developers when trying to learn new APIs through surveys and interviews with Microsoft developers. The study showed that many obstacles were related to aspects of the 
documentation, but did not include the systematic analysis of API documentation content.

Similarly, Shi et al. [8] studied API documentation evolution. The authors apply data mining techniques over the source repository of five open-source APIs. Their study provides various quantitative measures of which parts of the API documentation are most frequently revised, and how often API documentation is changed consistently with the corresponding elements.

\subsection{Knowledge Categorization based on Manual Methods}

Researchers have applied Knowledge from one field to other field, they also studied which are the different questions raised in Software Project Development.

Mylopoulos et al.[5] discussed how knowledge representation techniques from the field of Artificial Intelligence can be applied to software engineering. The authors presented a categorization of different knowledge types, presumably derived from their experience.

Requirement and Design are the important stages in Software Project Development. Herbsleb and Kuwana[4] classified questions asked in design meetings to study the kinds of knowledge that may benefit from explicit capture at the requirements and design stages based on their general experience.

Hou et al.[2] studied 300 questions related to two specific Swing widgets (JButton and JTree) posted on the Swing forum. They then mapped the questions to the different design features of the widgets. Their classification focuses more on the target of the question and less on discovering the different types of knowledge provided to and sought by API users.

More recently, Ko et al.[1] observed 17 developers at Microsoft for a 90 minutes session each, studying their information needs as they perform their software engineering tasks. From the observation data the authors collected 334 specific information needs, which they abstracted into 21 general information needs.

Kirk et al.[3] investigated the knowledge problems faced by them and their students when trying to develop applications by extending the JHotDraw framework.

Similarly to Ko et al.'s study, Sillito et al.[7] produced a catalog of 44 types of questions developers ask during software evolution tasks. The questions in the catalog do not focus exclusively on API usage, but rather relate to software evolution and maintenance tasks.

So, researchers focused on how different stages of Software Project Development and tools required can be analyzed in different ways and they classified the Questions raised in different phases into different categories based on their
Experience, Observations. Knowledge Types was not generated automatically.

Here, authors referred and studied API Reference Documentation in different ways. So, Separate study of Knowledge Categorization and API Reference Documentation was done previously.

The proposed work focuses on generation of Knowledge Types from Java API Reference Documentation, Classification and Searching in Java API Reference Documentation.

\section{IMPLEMENTATION DETAILS}

\subsection{Block Diagram of the System}

The following figure explains the Block Diagram of Proposed System:

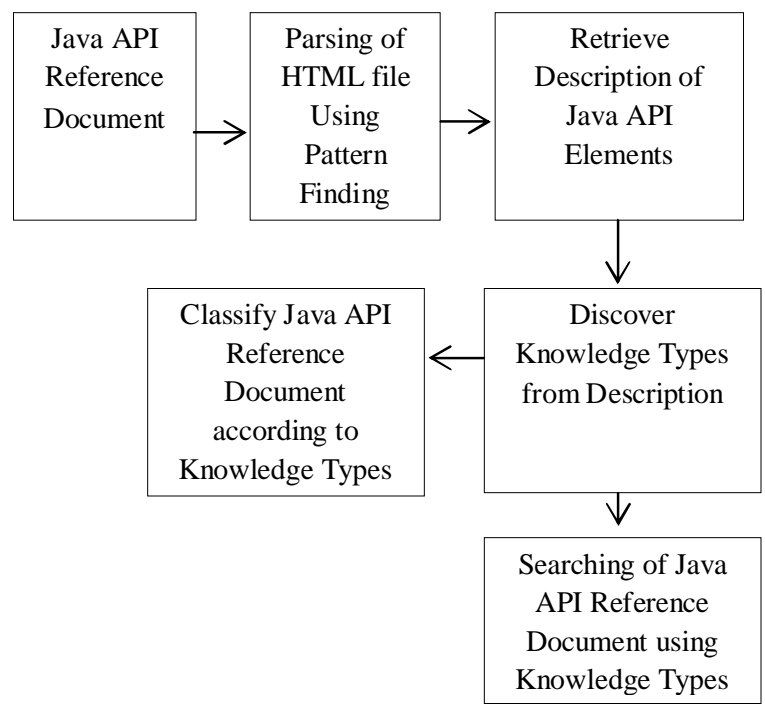

Figure 1: Block Diagram of the System

The System focuses on Java API Reference Documents that is Javadocs. Input to the system is API Reference Document of Java which is HTML Page.

This Java API Reference Document is then parsed by finding Pattern for the Tags having Description.

After finding the Patterns, the required Text is retrieved from the HTML page.

In the next step, Description of API elements is analyzed and then Knowledge Types will be generated.

Following are the Knowledge Types that are to be generated:

1. Functionality and Behavior: This Knowledge Type describes functionality and features of API. And also specifies what happens when the API is used.

e.g.: $\quad$ protected boolean enabled

Specifies whether action is enabled; the default is true.

2. Directives: It is related to accessibility that is what users are allowed or not allowed to do with the API element. Directives 
International Journal of Computer Applications Technology and Research

Volume 3- Issue 7, 451 - 456, 2014, ISSN: 2319-8656

are clear contracts.

e.g.: public class AccessException extends RemoteException

An AccessException is thrown by certain methods of the java.rmi.Naming class (specifically bind, rebind, and unbind) and methods of the java.rmi.activation.ActivationSystem interface to indicate that the caller does not have permission to perform the action requested by the method call. If the method was invoked from a non-local host, then an AccessException is thrown.

3. Control-Flow: How the API (or the framework) manages the flow of control is described by this knowledge type. For example by stating what events cause a certain callback to be triggered?

e.g.: $\quad$ Set $<$ String $>$ getSupportedAnnotationTypes()

If the processor class is annotated with SupportedAnnotationTypes, return an unmodifiable set with the same set of strings as the annotation.

4. Code Examples: Code examples are provided for how to use and combine elements to implement certain functionality or design outcomes.

e.g.: public abstract class AbstractExecutorService extends Object implements ExecutorService

Provides default implementations of ExecutorService execution methods. This class implements the submit, invokeAny and invokeAll methods using a RunnableFuture returned by newTaskFor, which defaults to the FutureTask. class provided in this package. For example, the implementation of submit(Runnable) creates an associated RunnableFuture that is executed and returned. Subclasses may override the newTaskFor methods to return RunnableFuture implementations other than FutureTask.

Extension example. Here is a sketch of a class that customizes ThreadPoolExecutor to use a CustomTask class instead of the default FutureTask:

public class CustomThreadPoolExecutor extends ThreadPoolExecutor \{

static class CustomTask $\langle\mathrm{V}\rangle$ implements RunnableFuture $<\mathrm{V}>\{\ldots\}$

protected $\quad\langle\mathrm{V}\rangle \quad$ RunnableFuture $\langle\mathrm{V}\rangle$

newTaskFor(Callable $<\mathrm{V}>\mathrm{c})\{$ return new CustomTask $<\mathrm{V}>(\mathrm{c})$;

\}

protected $\langle\mathrm{V}\rangle$ RunnableFuture $\langle\mathrm{V}\rangle$ newTaskFor(Runnable

$\mathrm{r}, \mathrm{V}$ v) \{

return new CustomTask $<\mathrm{V}>(\mathrm{r}, \mathrm{v})$

\}

// ... add constructors, etc.

\}

5. Environment: Aspects related to the environment in which the API is used is described in this type, but not the API directly, e.g., compatibility issues, differences between versions, or licensing information.

e.g: public abstract class AbstractElementVisitor7 $\langle\mathrm{R}, \mathrm{P}\rangle$ extends AbstractElementVisitor $6<\mathrm{R}, \mathrm{P}\rangle$

A skeletal visitor of program elements with default behavior appropriate for the RELEASE_7 source version.

6.External References: It includes any pointer to external documents, either in the form of hyperlinks, tagged "see also" reference, or mentions of other documents (such as standards

www.ijcat.com or manuals).

e.g: public interface DOMLocator

DOMLocator is an interface that describes a location (e.g. where an error occurred).

See also the Document Object Model (DOM) Level 3 Core Specification.

7. Non-information: A section of documentation containing any complete sentence or self-contained fragment of text that provides only uninformative boilerplate text.

e.g: DefinitionKindHelper()

After generating the Knowledge Types, the given Java API Reference Document is classified according to the Knowledge Types generated for class.

Also, searching of Document is done depending upon the Knowledge Types.

\subsection{Concept in detail with example:}

a) Consider following HTML file as Input: In this example, one of the class of Javadocs, named void AbstractAction is taken into consideration.

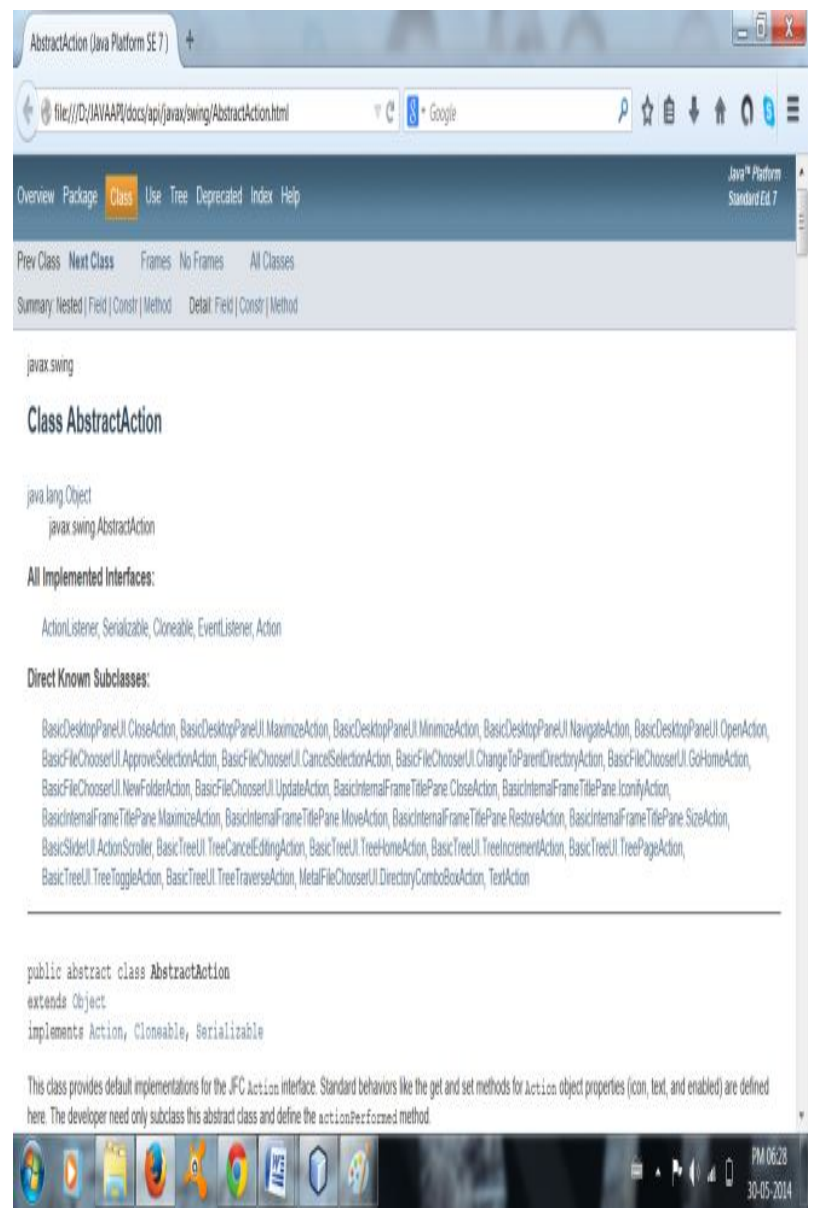

Figure 2: Example of the API Reference Document

b) Parsing of HTML document is done using following technique:

In this technique, initially all HTML Tags are fetched from 
the HTML Page.

After fetching all the HTML Tags, the Tags having the required descriptions are observed.

And then the required Text is retrieved from the all HTML Tags.

For Example: To get the Description of Class , all HTML Tags are observed.

And then Pattern is detected as: Description of the Class is present under div tag having identity as <div class="block" $\rangle$. But here, there will be multiple div tags in one HTML page with same class="block".

So again, pattern is observed in all HTML pages of Java API Reference Documents as: Description of the Class is always present in the First tag having class="block".

And then Text is retrieved from this tag.

So after this First div tag, multiple div tags with class= "block" may be present.

c) After separating the tags having the description, next step is to generate Knowledge Types for the given API Reference Document. Here description of one API elements of the API Reference Document may fall under more than one Knowledge Types.

To generate Knowledge Types, identity of each Knowledge Type is observed.

For Example:

For generating the Functionality and Behavior Knowledge Type, Description of API Elements is considered as it is. Because Functionality and Behavior Knowledge Type describes functionality and features of API. And also specifies what happens when the API is used.

For above class, Following Knowledge Types are generated: For Description of class: External References, Environment, Functionality and Behavior.

For Constructor of class: Functionality and Behavior.

For Fields, Methods of class: Control Flow, Functionality and Behavior.

d) After generating the Knowledge Types, depending upon the Knowledge Types generated for class the given Java API Reference Document is classified into respective Knowledge Type category.

So here for above example of AbstractAction, this document will be classified in External References, Environment ,Functionality and Behavior.

e) Knowledge Types generated will be used for searching. For above example of AbstractAction , user can search for "Code Example of AbstractAction" for getting Example of AbstractAction.

\subsection{Algorithms}

\subsubsection{Parsing of HTML Files to fetch Description:}

a) Initially, one of the Javadocs pages that is Java API Reference Document which is to be processed is taken as input.

b) Source code of the Javadocs is HTML tags and hence the actual input to the first step is HTML and JavaScript tags. c) After taking HTML tags as input, the next step is to parse the HTML tags to fetch the tags having description.

d) So, to fetch the description of API element from the current page using Pattern Finding.

\subsubsection{For Generation of Knowledge Types for the} Description of API elements:

a) After fetching the description of API elements in second step, next step is to process this description.

b) To process the description of the API elements, the patterns of the Descriptions are observed, that is whether the descriptions are having some common words in them or they are starting with same words or having some common format. c) So, after finding some common patterns in the descriptions, the Knowledge Types are generated.

That is description will be classified to the appropriate Knowledge Type. For Example, Description of all API elements will have common Knowledge Type as Functionality and Behaviour.

\subsubsection{For classification of Java API Reference Document:}

a) Here the Knowledge Types generated for Class are observed first.

b) Depending on the Knowledge Types generated for Class , the document will be classified into the respective Knowledge Types.

\subsubsection{Searching using Knowledge Types}

a) After classification of Documentation in above step, the searching will be performed.

b) Here depending upon query given by user, the query will be parsed and the searching of the query will be done depending upon the Knowledge Types.

\section{RESULTS}

\subsection{Data Set}

The Data Set for the system are set of API Reference Documents. The jdk-7u51-apidocs.zip file contains the set of API Reference Documents for Java. The above said file can be obtained by using following link:

http://www.oracle.com/technetwork/java/javase/documentatio n/java-se-7-doc-download-435117.html

\subsection{Results}

\subsubsection{Results of Classification:}

Consider the API Reference Documentation in Figure 2 for Class AbstractAction.

For API Reference Document in Figure 2, the document will be classified as follows:

Table 1: Results obtained for AbstractAction and for other Classes 
International Journal of Computer Applications Technology and Research

Volume 3- Issue 7, 451 - 456, 2014, ISSN: 2319-8656

\begin{tabular}{|c|c|c|c|}
\hline $\begin{array}{l}\text { Sr. } \\
\text { No. }\end{array}$ & Class Name & $\begin{array}{l}\text { Knowledge } \\
\text { Types } \\
\text { generated Of } \\
\text { Class }\end{array}$ & $\begin{array}{l}\text { Classification } \\
\text { of Document } \\
\text { Into Following } \\
\text { Knowledge } \\
\text { Types }\end{array}$ \\
\hline 1 & $\begin{array}{c}\text { AbstractActio } \\
\text { n (Java } \\
\text { Platform SE } 7 \\
\text { ) }\end{array}$ & $\begin{array}{c}\text { External } \\
\text { References, } \\
\text { Environment, } \\
\text { Functionaliy } \\
\text { and Behavior }\end{array}$ & $\begin{array}{c}\text { External } \\
\text { References } \\
\text {,Environment } \\
\text {,Functionaliy } \\
\text { and Behavior }\end{array}$ \\
\hline 2 & $\begin{array}{c}\text { AbstractAnno } \\
\text { tationValueVi } \\
\text { sitor6 (Java } \\
\text { Platform SE } 7 \\
\text { ) }\end{array}$ & $\begin{array}{l}\text { Environment, } \\
\text { Functionality } \\
\text { and Behavior }\end{array}$ & $\begin{array}{l}\text { Environment, } \\
\text { Functionality } \\
\text { and Behavior }\end{array}$ \\
\hline 3 & $\begin{array}{c}\text { BoxLayout } \\
\text { (Java Platform } \\
\text { SE } 7 \text { ) }\end{array}$ & $\begin{array}{c}\text { External } \\
\text { References, } \\
\text { Environment, } \\
\text { Control Flow, } \\
\text { Functionality } \\
\text { and Behavior }\end{array}$ & $\begin{array}{c}\text { External } \\
\text { References, } \\
\text { Environment, } \\
\text { Control Flow, } \\
\text { Functionality } \\
\text { and Behavior }\end{array}$ \\
\hline 4 & $\begin{array}{c}\text { ButtonGroup } \\
\text { (Java Platform } \\
\text { SE } 7 \text { ) }\end{array}$ & $\begin{array}{c}\text { External } \\
\text { ReferencesEn } \\
\text { vironmentFun } \\
\text { ctionality and } \\
\text { Behavior }\end{array}$ & $\begin{array}{c}\text { External } \\
\text { ReferencesEnvir } \\
\text { onmentFunction } \\
\text { ality and } \\
\text { Behavior }\end{array}$ \\
\hline 5 & $\begin{array}{c}\text { CellRenderer } \\
\text { Pane (Java } \\
\text { Platform SE } 7 \\
\text { ) }\end{array}$ & $\begin{array}{c}\text { External } \\
\text { ReferencesCo } \\
\text { de } \\
\text { ExampleEnvir } \\
\text { onmentFuncti } \\
\text { onality and } \\
\text { Behavior }\end{array}$ & $\begin{array}{c}\text { External } \\
\text { ReferencesCode } \\
\text { ExampleEnviro } \\
\text { nmentFunctiona } \\
\text { lity and } \\
\text { Behavior }\end{array}$ \\
\hline 6 & $\begin{array}{c}\text { DefaultButton } \\
\text { Model (Java } \\
\text { Platform SE } 7 \\
\text { ) }\end{array}$ & $\begin{array}{c}\text { External } \\
\text { ReferencesEn } \\
\text { vironmentFun } \\
\text { ctionality and } \\
\text { Behavior }\end{array}$ & $\begin{array}{c}\text { External } \\
\text { ReferencesEnvir } \\
\text { onmentFunction } \\
\text { ality and } \\
\text { Behavior }\end{array}$ \\
\hline 7 & $\begin{array}{l}\text { DefaultListCe } \\
\text { llRenderer } \\
\text { (Java Platform } \\
\text { SE 7) }\end{array}$ & $\begin{array}{c}\text { External } \\
\text { ReferencesEn } \\
\text { vironmentCon } \\
\text { trol } \\
\text { FlowFunction } \\
\text { ality and } \\
\text { Behavior }\end{array}$ & $\begin{array}{c}\text { External } \\
\text { ReferencesEnvir } \\
\text { onmentControl } \\
\text { FlowFunctionali } \\
\text { ty and Behavior }\end{array}$ \\
\hline 8 & $\begin{array}{c}\text { DefaultRowS } \\
\text { orter (Java } \\
\text { Platform SE } 7 \\
\text { ) }\end{array}$ & $\begin{array}{c}\text { External } \\
\text { ReferencesCo } \\
\text { ntrol } \\
\text { FlowFunction } \\
\text { ality and } \\
\text { Behavior }\end{array}$ & $\begin{array}{c}\text { External } \\
\text { ReferencesCont } \\
\text { rol } \\
\text { FlowFunctionali } \\
\text { ty and Behavior }\end{array}$ \\
\hline
\end{tabular}

\subsubsection{Results of Searching:}

When user wish to search query like "Code Examples of AbstractAction" the results will show that respective document.

So depending upon user requirement the Searching is done.

\subsubsection{Performance Measure:}

Following table shows Performance Measures:

Table 2: Performance Measure for different documents tested by system

\begin{tabular}{|c|c|c|c|}
\hline $\begin{array}{c}\text { Java API } \\
\text { Reference } \\
\text { Document } \\
\text { Tested }\end{array}$ & $\begin{array}{c}\text { Knowledge } \\
\text { Types } \\
\text { Expected }\end{array}$ & $\begin{array}{c}\text { Knowledge } \\
\text { Types } \\
\text { Generated }\end{array}$ & Precision \\
\hline $\begin{array}{l}\text { AbstractAction } \\
\text { (Java Platform } \\
\text { SE 7 ) }\end{array}$ & 4 & 4 & 1.00 \\
\hline $\begin{array}{l}\text { AbstractAnnotat } \\
\text { ionValueVisitor } \\
6 \text { (Java Platform } \\
\text { SE 7) }\end{array}$ & 2 & 2 & 1.00 \\
\hline $\begin{array}{l}\text { BoxLayout } \\
\text { (Java Platform } \\
\text { SE 7 ) }\end{array}$ & 4 & 4 & 1.00 \\
\hline $\begin{array}{l}\text { ButtonGroup } \\
\text { (Java Platform } \\
\text { SE 7 ) }\end{array}$ & 4 & 3 & 0.75 \\
\hline $\begin{array}{l}\text { CellRendererPa } \\
\text { ne (Java } \\
\text { Platform SE } 7 \text { ) }\end{array}$ & 6 & 5 & 0.83 \\
\hline $\begin{array}{l}\text { DefaultButtonM } \\
\text { odel (Java } \\
\text { Platform SE } 7 \text { ) }\end{array}$ & 3 & 3 & 1.00 \\
\hline $\begin{array}{l}\text { DefaultListCell } \\
\text { Renderer (Java } \\
\text { Platform SE } 7 \text { ) }\end{array}$ & 5 & 4 & 0.80 \\
\hline $\begin{array}{l}\text { DefaultRowSort } \\
\text { er (Java } \\
\text { Platform SE } 7 \text { ) }\end{array}$ & 3 & 3 & 1.00 \\
\hline $\begin{array}{l}\text { GroupLayout } \\
\text { (Java Platform } \\
\text { SE 7 ) }\end{array}$ & 3 & 3 & 1.00 \\
\hline $\begin{array}{l}\text { ImageIcon (Java } \\
\text { Platform SE } 7 \text { ) }\end{array}$ & 4 & 3 & 0.75 \\
\hline
\end{tabular}




\section{CONCLUSION AND FUTURE WORK}

API's are used as interface for using predefine functions, packages, classes etc. Developers read API reference documentation to learn how to use the API and answer specific questions they have during development tasks. Thus API Reference Documentation provides guide to user for referring to API. API Reference Documentation contains description of API elements; this description will be analyzed for generating Knowledge. This system focuses on classification of description of API elements into different Knowledge Types for Java API Reference Documentation. After generating Knowledge Types, classification of the Java API Reference Document is done according to Knowledge Types. Java API Reference Document then can be searched using the Knowledge Types. Other Types of Documentation like MSDN, Documentation of Python can be considered for parsing and processing further.

\section{REFERENCES}

[1] A. J. Ko, R. DeLine, and G. Venolia, "Information needs in collocated software development teams," in Proceedings of the 29th International Conference on Software Engineering, 2007, pp. 344-353.

[2] D. Hou, K. Wong, and J. H. Hoover, "What can programmer questions tell us about frameworks?" in Proceedings of the 13th International Workshop on Program Comprehension, 2005, pp. 87-96.

[3] D. Kirk, M. Roper, and M. Wood, "Identifying and addressing problems in object-oriented framework reuse," Empirical Software Engineering, vol. 12, pp. 243-274, June 2007.

[4] J. D. Herbsleb and E. Kuwana, "Preserving knowledge in design projects: what designers need to know," in Proceedings of the Joint INTERACT '93 and CHI '93 Conferences on Human Factors in Computing Systems, 1993, pp. 7-14.

[5] J. Mylopoulos, A. Borgida, and E. Yu, "Representing software engineering knowledge," Automated Software Engineering, vol. 4, no. 3, pp. 291-317, 1997.

[6] J. Nykaza, R. Messinger, F. Boehme, C. L. Norman, M. Mace,and M. Gordon, "What programmers really want: Results of a needs assessment for SDK documentation," in Proceedings of the 20th Annual ACM SIGDOC International Conference on Computer Documentation, 2002, pp. 133-141.

[7] J. Sillito, G. C. Murphy, and K. D. Volder, "Asking and answering questions during a programming change task," IEEE Transactions on Software Engineering, vol. 34, no. 4, pp. 434-451, July-August 2008.

[8] L. Shi, H. Zhong, T. Xie, and M. Li, "An empirical study on evolution of API documentation," in Proceedings of the Conference on Fundamental Approaches to Software Engineering, 2011, pp. 416-431.

[9] M. P. Robillard and R. DeLine, "A field study of API learning obstacles," Empirical Software Engineering, vol. 16, no. 6, pp. 703-732, 2011.

[10] S. Y. Jeong, Y. Xie, J. Beaton, B. A. Myers, J. Stylos, R. Ehret, J. Karstens, A. Efeoglu, and D. K. Busse, "Improving documentation for eSOA APIs through user studies," in Proc. 2nd Int'l Symp. on End-User Development, ser. LNCS, vol. 5435. Springer,2009, pp. 86-105.

[11] Walid Maalej and Martin P. Robillard , Patterns of Knowledge in API Reference Documentation, IEEE TRANSACTIONS ON SOFTWARE ENGINEERING, VOL. 39, NO. X, XXXXXXX 2013. 